\title{
APLICAÇÃO FOLIAR DE CÁLCIO E BORO COM PRODUTO INDUSTRIALIZADO PARA AVALIAÇÃO DE REDIMENTO E QUALIDADE DE SEMENTE DE SOJA
}

ARANTES, Rafael Pereira ${ }^{1}$
CURY, Tadeu Nascimento ${ }^{2}$
LEÃO, Fabiana de Paula ${ }^{3}$
MACIEL JUNIOR, Vinicius Antonio ${ }^{4}$

Recebido em: 2009-05-06

Aprovado em: 2009-09-29

ISSUE DOI: $10.3738 / 1982.2278 .205$

RESUMO: O presente experimento foi em condições de campo, com a cultivar de soja BRS Valiosa $\mathrm{RR}$, com aplicação do produtos $\mathrm{CaB}_{2}$ (composição de $\mathrm{Ca}-10 \%$ p/p, B - 2\% p/p) em diferentes fases de desenvolvimento da cultura no ano agrícola 07/08, no município de Ituverava - SP.O experimento constituiu de oitos parcelas sendo: pré-florada, pré-florada + floração, pré-florada + floração + vagem, pré - floração + vagem, floração, floração + vagem, vagem e testemunha, utilizado o delineamento experimental bloco ao acaso com 8 tratamentos e 4 repetições por parcelas. O objetivo do trabalho foi analisar qual a fase de desenvolvimento e dosagem são mais adequados para aplicação de produtos à base de cálcio e boro na cultura da soja. Foram realizadas avaliações dos números de grão por vagem, peso de 100 sementes e número de vagem onde observou-se que a diferenças de dosagens e fase de desenvolvimento da cultura não mostraram diferenças significativas para aplicação destes produtos.

Palavras - chaves: Soja. Plantio direto. Adubação foliar

\section{FOLIAR APPLICATION OF CALCIUM AND BORON INDUSTRIALIZED PRODUCTS COM FOR EVALUATION OF QUALITY ANDA INCOME OF SOYBEAN SEEDS}

\begin{abstract}
SUMMARY: This experiment was in the field with the soybean cultivar BRS Valuable RR, applying the product $\mathrm{CaB}_{2}$ (composition of $\mathrm{Ca}-10 \% \mathrm{w} / \mathrm{w}, \mathrm{B}-2 \% \mathrm{w} / \mathrm{w}$ ) at different stages of development of culture in season 07/08 in the town of Ituverava - SP. The experiment consisted of eight plots being: prebloom, pre-bloom + flowering, pre-bloom + flowering + pod, pre - flowering + pod, flowering, flowering + pod, pods and control, using randomized block experimental design with 8 treatments and 4 replicates perplot. The objective was to analyze which stage of development and strength are more suitable for application of products based on calcium and boron in the soybean crop. Was evaluated in the number of grains per pod, 100 seed weight and number of pods where it was observed that the differences in strengths and development stage of the culture showed no significant differences in application of these products.
\end{abstract}

Keywords: Soybean. Tillage. Foliar fertilization

\footnotetext{
${ }^{1}$ Acadêmico do curso de Agronomia da Faculdade Dr. Francisco Maeda da Fundação Educacional de Ituverava/FEI. Rodovia Jerônimo Nunes Macedo km 1, CEP 14500-000, Ituverava-SP.

${ }^{2}$ Engenheiro Agrônomo pela Faculdade Dr. Francisco Maeda da Fundação Educacional de Ituverava/FEI.

Rodovia Jerônimo Nunes Macedo km 1, CEP 14500-000, Ituverava-SP.

${ }^{3}$ Engenheira de Alimentos pela Universidade Federal de Viçosa-MG

${ }^{4}$ Mestre em Agronomia, Coordenador do Curso de Agronomia da FE/FAFRAM.
} 


\section{INTRODUÇÃO}

A soja (Glycine $\max (L)$ merril) e uma oleaginosa originaria do sudoeste Asiático, com grande utilização na alimentação humana e animal, por se tratar de uma planta que produz grãos ricos em proteínas. Considerando que sua proteína e de elevada qualidade e de baixo custo, a cultura assume um papel de grande importância na solução de alimentação dos povos. (MARCONDES et al , 1981).

Cerca de $90 \%$ da massa das plantas está constituída por carbono, hidrogênio e oxigênio, elementos obtêm do ar e água. Os 10\% restante que correspondem aos demais elementos, constituem a denominada fração mineral.

Segundo proposto por Arnon; Stout, os elementos essenciais a se aplica a todo que são necessários para o desenvolvimento e reprodução das plantas. Uma planta só pode completar seu ciclo, se lhe fornece em quantidade suficiente, todos os elementos minerais que lhe são essenciais. Os nutrientes essenciais obedecem a lei de shelford: assim como algum nutriente encontra-se mais próximo do mínimo critico necessário, outro nutriente pode atingir concentrações acima das necessárias no tecido e causar sintomas de toxidez na planta.

Os baixos teores de matéria orgânica no solo realçam a deficiência de micronutriente no solo como: boro(B), zinco $(\mathrm{Zn})$, molibdênio $(\mathrm{Mo})$ e cobre $(\mathrm{Cu})$ (EMBRAPA, 1999). A carência de boro e muito comum no país, particularmente em solos arenosos e pobres em matéria orgânica (MALAVOLTA, 1985)

$\mathrm{O}$ elemento químico $\mathrm{Ca}$, assim como o $\mathrm{B}$, são imóveis no floema e não se redistribuem na planta, assim a deficiência nutricional de ambos se apresenta em órgãos mais novos ( MALAVOLTA, 1976,1985) As principais funções do Ca na planta são: atuar na formação do pectato de cálcio, presente na lamela media da parede celular e na germinação do grão de pólen e crescimento do tubo polínico. O B e importante na translocação de açúcares e formação da parede celular. Para Rosolem et al.(1990), o cálcio atua decisivamente no numero de flores e vagens abortadas em soja, e existe alta correlação negativa entre o teor de Ca na planta e numero de flores e vagens abortada. Há relatos de aumento da produtividade de algumas culturas com o uso de bórax e outros micronutrientes.

A adubação foliar, porém, não deve ser encarada como substituição da adubação radicular e sim como um complemento a esta.

Assim, torna - se essencial o seu estudo para avaliar suas resposta e vantagens na produção agrícola. 
Nesse trabalho instalado na fazenda estiva, no município de Ituverava-SP, estudou-se a resposta a adubação foliar de Ca e B com diferentes dosagem, produto e fase desenvolvimento, captando assim a realidade do agricultor que pode realizar com dosagem e fases de desenvolvimento ideal.

O objetivo deste experimento foi avaliar a influencia da adubação foliar através de Ca e B na produção de soja com diferentes produtos e fases de desenvolvimento.

\section{MATERIAL E MÉTODO}

O trabalho foi realizado na fazenda Estiva, no município de Ituverava - SP, onde a altitude e de $631 \mathrm{~m}$, e a precipitação durante o trabalho foi de $1325 \mathrm{~mm}$ e foi utilizado a cultivar de soja BRS Valiosa RR em plantio direto em palha de cana-de-açúcar.

Os tratamentos foram delineados em blocos ao acaso, com 8 tratamento e 4 repetições de cada parcela selecionada, no qual utilizou-se o testes de Tukey a 5\%.

Cada parcela constituiu de 6 linhas de espaçamento de $0,50 \mathrm{~cm}$, sendo que a área total de cada parcela era de $12 \mathrm{~m}^{2}$, sendo que a área utilizada para avaliação foi somente $8 \mathrm{~m}^{2}$.

Nos tratamentos utilizou o produto $\mathrm{CaB}_{2}$ (composição de $\mathrm{Ca}-10 \%$ p/p, B-2\% p/p), com aplicação em várias etapas do desenvolvimento da cultura, para avaliação da época mais recomendada de aplicação para aumento de rendimento da soja.

Os tratamentos com o adubo foliar estão relacionados na Tabela 01, os quais estão demonstrados as datas de suas aplicações e dosagens.

TABELA 1: Dados da aplicação.

(Continua)

\begin{tabular}{|c|c|}
\hline \multicolumn{2}{|c|}{ Data de Aplicação } \\
\hline $16 / 01 / 2008$ & $\begin{array}{l}\text { Pré -floração } \\
\text { - } \quad \text { Pré-florada (Industrializado) } 27 \mathrm{ml} \mathrm{de} \mathrm{CaB2} \\
\text { - } \quad \text { Pré-florada + florada + vagem (Industrializado) } 9 \mathrm{ml} \mathrm{de} \mathrm{CaB2} \\
\text { - } \quad \text { Pré-florada + florada (Industrializado) } 13.5 \mathrm{ml} \mathrm{de} \mathrm{CaB2} \\
\text { - } \quad \text { Pré-florada + vagem (Industrializado) } 13.5 \mathrm{ml} \mathrm{de} \mathrm{CaB2}\end{array}$ \\
\hline $01 / 02 / 2008$ & $\begin{array}{l}\text { Floração plena (80\%) } \\
\text { - Florada (Industrializado) } 27 \mathrm{ml} \mathrm{de} \mathrm{CaB2} \\
\text { - Pré-florada + florada + vagem (Industrializado) } 9 \mathrm{ml} \mathrm{de} \mathrm{CaB2} \\
\text { - Pré-florada + florada (Industrializado) } 13.5 \mathrm{ml} \mathrm{de} \mathrm{CaB2} \\
\text { - }\end{array}$ \\
\hline
\end{tabular}


TABELA 1: Dados da aplicação.

(Conclusão)

28/02/2008 Vagem com 3 a 5 mm

- Vagem (Industrializado) $27 \mathrm{ml} \mathrm{de} \mathrm{CaB2}$

- Pré-florada + florada + vagem (Industrializado) $9 \mathrm{ml} \mathrm{de} \mathrm{CaB} 2$

- Florada + vagem (Industrializado) $13.5 \mathrm{ml} \mathrm{de} \mathrm{CaB} 2$

- Pré-florada + vagem (Industrializado) $13.5 \mathrm{ml} \mathrm{de} \mathrm{CaB2}$

Foram realizados aplicações com pulverizador costal de 20 litros, utilizado bico tipo cone da serie TT - 110 - 03 com vazão de 200 litro/ha mantido a pressão constante por válvula de pressão constante.

O plantio da cultivar foi realizado no dia 20/11/2207 com semeadora de plantio direto em solo classificado anteriormente como latossolo vermelho distroferrico, textura argilosa amoderado. O espaçamento foi de $0,50 \mathrm{~cm}$ entre linha com densidade de 300.000 sementes por metro linear, chegando a stand final de 260.000 plantas por metro linear.

No plantio utilizou adubação calculada baseada nos teores encontrados na análise de solo, para uma produtividade de 3 toneladas por hectares e a quantidade utilizada foi de $250 \mathrm{~kg} / \mathrm{h}$ dá fórmula 04-20-20. Aos 30 dias após o plantio foi feito uma cobertura a lanço com $25 \mathrm{~kg} / \mathrm{ha}$ de $\mathrm{KCl}(60 \% \mathrm{~K} 2 \mathrm{O})$

Realizou-se a colheita no dia 26/04/2008, e o processo da colheita foi manual com arranquio de 10 plantas das duas linhas centrais da parcela, descartando as linhas laterais.

Foram avaliados o número de vagens; número de grão por vagem, peso de 100 sementes onde foi retirado a umidade e corrigida para $14 \%$.

\section{RESULTADO E DISCUSSÃO}

Os tratamentos realizados em condições de campo no município de Ituverava não mostraram resultados significativos para utilização de produtos a base de cálcio e boro. Os dados estão na tabela 02: 
TABELA 2: Analise de variação do experimento

\begin{tabular}{lccc}
\hline \multicolumn{1}{c}{ Tratamento } & $\begin{array}{l}\text { Número de } \\
\text { vagem }\end{array}$ & $\begin{array}{c}\text { Número de } \\
\text { grãos por vagens }\end{array}$ & $\begin{array}{c}\text { Peso de 100 } \\
\text { sementes }(\mathbf{g})\end{array}$ \\
\cline { 2 - 3 } & $63.4750 \mathrm{a}$ & $123.5000 \mathrm{a}$ & $3.0500 \mathrm{a}$ \\
Pré florada + florada & $61.0000 \mathrm{a}$ & $142.9250 \mathrm{a}$ & $2.5750 \mathrm{a}$ \\
Pré florada + vagem & $52.8250 \mathrm{a}$ & $100.4750 \mathrm{a}$ & $2.6250 \mathrm{a}$ \\
Pré florada + florada + vagem & $56.3000 \mathrm{a}$ & $104.6500 \mathrm{a}$ & $3.2250 \mathrm{a}$ \\
Florada & $50.6500 \mathrm{a}$ & $100.8500 \mathrm{a}$ & $1.6000 \mathrm{a}$ \\
Florada + vagem & $54.4250 \mathrm{a}$ & $105.7000 \mathrm{a}$ & $2.0500 \mathrm{a}$ \\
Vagem & $59.4000 \mathrm{a}$ & $119.8500 \mathrm{a}$ & $2.4500 \mathrm{a}$ \\
Testemunha & $58.6000 \mathrm{a}$ & $114.6250 \mathrm{a}$ & $2.9000 \mathrm{a}$ \\
\hline
\end{tabular}

Analisando os resultados dispostos na Tabela, observou-se que não houve diferenças significativas para numero de vagens, numero de semente por vagem e peso de 100 sementes.

Segundo Kappes et. al. 2007 que também trabalhou com adubação foliar de cálcio e boro que verificaram e constataram o resultados significativos para aplicação na cultura da soja.

Neste experimento os resultados não mostraram vantagens em alterar a época de aplicação e fases de desenvolvimento.

\section{CONCLUSÃO}

As condições de aplicação em diferentes fases de desenvolvimento da cultura não apresentaram resultados significativos. Sendo não justificativos o uso de produtos a base de cálcio e boro via foliar.

\section{REFERÊNCIAS}

ARAUJO, S.A et al. Cultura do feijoeiro comum no Brasil. Piracicaba: Potafos, 1996.

BEVILAQUA, G. A. P.; SILVA FILHO, P. M.; POSSENTI, J. C. Aplicação foliar de cálcio e boro e componentes de rendimento e qualidade de sementes de soja. Cienc. Rural, Santa Maria, v. 32, n. 1, 2002.

BOARETTO, A. E. et al. Absorção foliar e translocação de cálcio pelo feijoeiro. Científico, São Paulo, v.11, p 227-237. 1983

CASTRO P.R.C.; KLUGE, R. A.; PERES, L. E. P. Manual de fisiologia vegetal. Piracicaba: Agronômica Ceres, 2005. 
CAMARGO, P. N.; SILVA, O. Manual de adubação foliar. São Paulo: Herba, 1975.p. 210

EMBRAPA, Empresa Brasileira de Pesquisa Agropecuária. Centro Nacional de Pesquisa de Soja. Recomendação técnicas para a cultura de soja no Paraná 1999/2000. Londrina, 1999. 236p. (Embrapa soja. Documento, 131).

MALAVOLTA, E. Manual de química agrícola. São Paulo: Agronômica Ceres, 1976. 528p.

MALVOLTA, E. Nutrição de plantas. Fisiologia vegetal. São Paulo: EDUSP, 1985. V. 1, 400 p.

MALAVOLTA, E. Manual de calagem e adubação das principais culturas. São Paulo: Agronomia Ceres, 1987.

ROSOLEM, C.A.; BOARETTO, A. E.; NAKAGAMA, J. Adubação foliar do feijoeiro. VIII. Fontes e doses de cálcio. Cientifica, São Paulo, v.18, p. 9-14. 1991 